\title{
THz Driven Accelerators and X-ray Sources
}

\author{
Franz X. Kärtner \\ Center for Free-Electron Laser Science and Deutsches Elektronen-Synchrotron (DESY), Notkestraße 85, 22607 Hamburg, Germany \\ Department of Physics and Center for Ultrafast Imaging, University of Hamburg, Luruper Chaussee 149, 22761 Hamburg, Germany \\ e-mail address: franz.kaertner@desy.de
}

\begin{abstract}
Approaches towards a linear THz accelerator technology are discussed. Theoretical and first experimental results on laser based high energy $\mathrm{THz}$ generation, guns, accelerators as well as compact X-ray sources based on these devices are presented.

OCIS codes: (32ß.0320) Ultrafast Optics; (190.4410) Nonlinear optics, parametric processes; (190.7110) Ultrafast nonlinear optics; (160.3730) Lithium Niobate; (320.7090) Ultrafast lasers.
\end{abstract}

\section{Introduction}

Today, high brightness and highly relativistic electron beams are generated by circular or linear accelerators (LINAC) typically operating with 1-3 GHz accelerating frequencies and approaches towards X-band frequencies in the $10 \mathrm{GHz}$ range are maturing. The achievable accelerating gradients are limited by field emission from cavity walls influenced by pulsed heating to several tens of $\mathrm{MV} / \mathrm{m}$ in the case of low frequencies and up to $100 \mathrm{MV} / \mathrm{m}$ in the case of X-band frequencies. Short electron bunches are typically created by photoemission from the cathode in the presence of a strongly accelerating field followed by bunch compression. Low charge bunches, 1-10 pC, may be compressed to durations down to $3-10 \mu \mathrm{m}$ (or $10-30 \mathrm{fs}$ in pulse duration). At a given accelerating field strength and RF frequency, compression is limited by space charge. With higher operating frequency, higher gradients in the accelerating electric field arise which enhances velocity bunching and leads to the generation of shorter electron bunches. These short electron bunches can be used for ultrafast electron diffraction or intense X-ray production. Choosing an operating frequency of the accelerator in the THz range, i.e. here $0.1-0.5 \mathrm{THz}$ and using multi-cycle to single-cycle pulses, accelerating fields in the 0.3 to $1 \mathrm{GV} / \mathrm{m}$ range are sustainable and bunch compression to the sub-femtosecond level of significant charge on the order of $\sim 1 \mathrm{pC}$ becomes possible. To drive electron guns and accelerators high-energy single-cycle and multi-cylce THz pulses in the $\mathrm{mJ}$ and tens of $\mathrm{mJ}$ range, respectively, are necessary. Here, we discuss approaches towards this goal and results achieved sofar.

\section{High energy THz generation}

The traditional approach towards generation of single-cycle $\mathrm{THz}$ pulses is via optical rectification in Lithium Niobate using the tilted pulse front technique (TPFT) [1]. Using 1 micron, sub-ps pulses up to $0.4 \mathrm{~mJ} \mathrm{THz}$ pulses have been generated with up to $0.8 \%$ efficiency albeit the center frequency moved below $0.2 \mathrm{THz}$, which would then need even higher $\mathrm{THz}$ energies as a driver, since corresponding structures are larger [2]. Here, we report generation of single-cycle THz pulses with a center frequency of $0.28 \mathrm{THz}$, an energy of $0.2 \mathrm{~mJ}$ and a conversion efficiency of $0.5 \%$ using $40-\mathrm{mJ}, 1020-\mathrm{nm}, 1-\mathrm{ps}$ pulses from a cryogenic Yb:YLF laser generated with the TPFT method in cryogenically cooled Lithium Niobate. Numerical simulations show, that further scaling to $1 \%$ efficiency with 100 $\mathrm{mJ}$ driver pulses is possible and should result in $1 \mathrm{~mJ} \mathrm{THz}$ pulses at about $0.3 \mathrm{THz}$ wavelength. Cryogenic cooling of Lithium Niobate lowers the absorption at $\mathrm{THz}$ frequencies. The conversion efficiency is limited due to break-up of the driver pulses after excessive cascading [3].

For multi-cycle or narrowband $\mathrm{THz}$ generation, difference frequency generation (DFG) in cryogenically cooled periodically poled lithium niobate (PPLN) crystals using quasi-phase matching promises high conversion efficiency of several percent. Cryogenic cooling allows the use of long crystals and cascading does not lead to break-up of the driver pulses as in the TPFT case due to collinear interaction of the waves [4]. DFG using highenergy broadband Ti:Sappire pulses and the chirp and delay method to match the difference frequency to the quasiphase matching period resulted in $40 \mu \mathrm{J}$ pulses at $0.5 \mathrm{THz}$ and $260 \mathrm{ps}$ duration [5]. The use of large aperture crystals fabricated by the Taira group and linearization of the difference frequency between the chirped and delayed pulses lead to further improvements by more than an order of magnitude to produce $460 \mu \mathrm{J}$ of $\mathrm{THz}$ radiation with about $460 \mathrm{~mJ}$ of laser pulses [6]. The future goal is to generate with $5 \%$ conversion efficiency up to $50 \mathrm{~mJ}$ of multicylce $\mathrm{THz}$ pulses from 1-J laser pulses to drive a THz accelerator using dielectrically loaded metal waveguides [7].

\section{THz guns and acceleration}

Over the last years, demonstrations have shown the feasibility of a $\mathrm{THz}$ accelerator technology. A proof of principle $\mathrm{THz}$ acceleration experiment based on laser-generated single-cycle $\mathrm{THz}$ pulses with $10 \mu \mathrm{J}$ pulse energy at $0.45 \mathrm{THz}$ has been performed recently [8]. First $\mathrm{THz}$ guns based on a parallel plate $\mathrm{THz}$ waveguide have been reported 
producing quasi-monochromatic electron bunches with a few percent energy spread around $400 \mathrm{eV}$ mean energy [9]. More advanced $\mathrm{THz}$ gun structures driven by either single-cycle or multi-cycle $\mathrm{THz}$ pulses have been proposed $[10,11]$. The single-cycle gun structure is a segmented $\mathrm{THz}$ waveguide device composed of arrays of parallel plate waveguides with dielectric fillings to delay the $\mathrm{THz}$ pulses in each waveguide, such that when excited with a single high energy $\mathrm{THz}$ pulse jointly, a tilted pulse front is created at the interaction point with electrons. This leads to
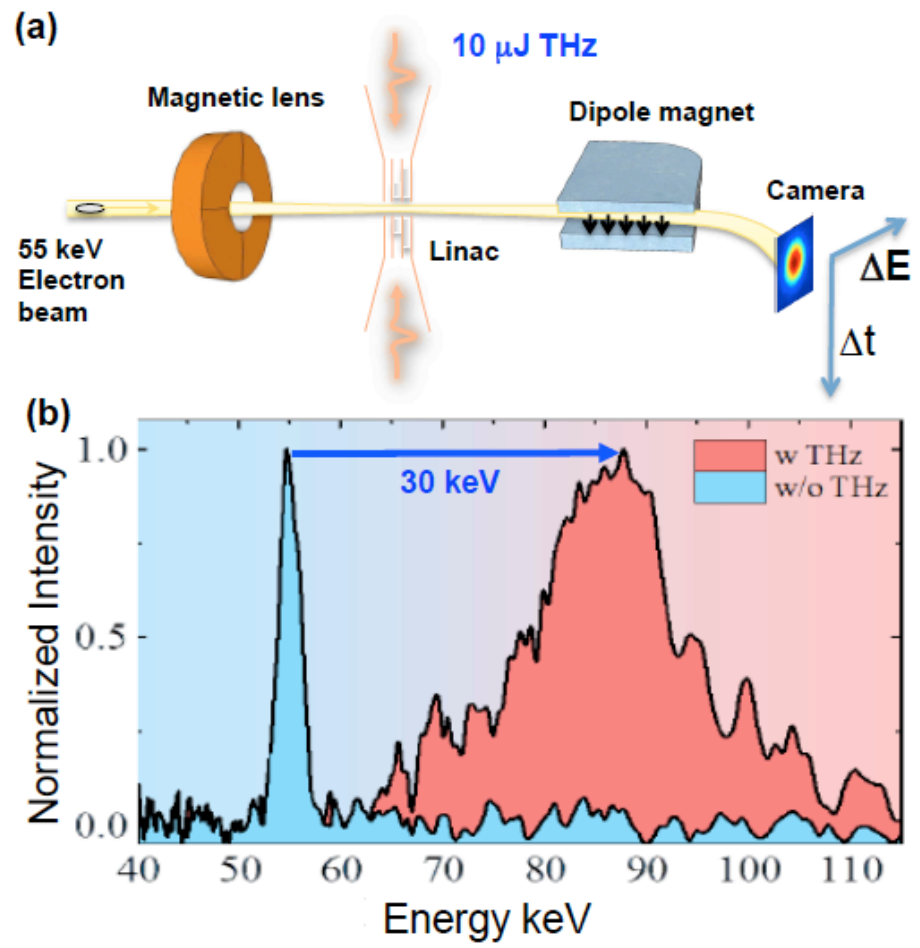

Fig. 1: (a) Acceleration of an electron bunch from a $55 \mathrm{keV}$ DC-gun using a segmented $\mathrm{THz}$ waveguide acceleration device with dipole magnet for energy measurement on MCP; (b) electron energy spectrum without (black) and with THz pulse(red). [12] continuous acceleration of the electron bunch in the interaction areas, see Fig. 1 (a). To demonstrate this single cycle accelerator concept, we used a UV-photo-triggered, 55 $\mathrm{kV}$ DC-gun to inject an electron bunch into the accelerating structure. The accelerating structure is driven by two single-cycle $\mathrm{THz}$ pulses with about $10 \mu \mathrm{J}$ of THz energy. Fig. 1 (b) shows the energy spectrum of the accelerated electron bunch, measured by a dipole magnet and an MCP. The spectrum measured with and without the $\mathrm{THz}$ pulses are shown. The $\mathrm{THz}$ accelerator boosts the average electron energy by $35 \mathrm{keV}$ and significantly broadens the energy distribution due to the long electron bunch from the DCgun of about 500 fs. The long pulse fills significant portion of the about 3 ps period accelerating $\mathrm{THz}$ field cylce. For acceleration, the two $\mathrm{THz}$ pulses are injected such that the electric fields constructively interfere in the center of the structure, and, in forward direction The transvers magnetic fields are canceled. If the pulses are delayed with respect to each other by half a $\mathrm{THz}$ wavelength, the electric fields cancel in the center of the device and the transvers magnetic fields add, which leads to a transvers deflection of the electron bunch.

This operation mode can be used for streaking of the electron bunch to measure its duration [12]. The deflection strength of about $140 \mu \mathrm{rad} / \mathrm{fs}$ allows for sub-10 femtosecond resolution. The structure has been also used to demonstrate temporal pulse compression, focussing and defocussing with up to $2 \mathrm{kT} / \mathrm{m}$ focussing strength.

Currrent work is geared towards pushing laser based $\mathrm{THz}$ generation to the multi-mJ level using Joule level, picosecond laser pulses with optimized pulse formats [4]. Such high energy $\mathrm{THz}$ pulses can accelerate electrons to the $10-20 \mathrm{MeV}$ level, energetic enough to enable in a first step hard X-ray generation via inverse Compton scattering [13].

\section{References}

[1] J. Hebling, G. Almasi, I. Z. Kozma, J. Kuhl, Opt. Exp. 10, 1161 (2012).

[2] J. A. Fülop, Z. Ollmann, C. Lombosi, C. Skrobol, S. P. Klingebiel, L. Palfalvi, F. Krausz, S. Karsch, J. Hebling, Opt. Exp. 22 , 20155 (2014).

[3] K. Ravi, W. R. Huang, S. Carbajo, X. Wu and F. X. Kärtner, Opt. Exp. 22, 20239 (2014).

[4] K. Ravi, et al. Opt. Lett. 41, 3806 (2016) and Opt. Lett. 42, pp. 25582 (2016).

[5] F. Ahr, S. W. Jolly, N. H. Matlis, S. Carbajo. T. Kroh, K. Ravi, D. N. Schimpf, J. Schulte, H. Ishizuki, T. Taira, A. Maier and F. X. Kärtner, Opt. Lett. 42, 2118 (2017).

[6] S. W. Jolly, F. Ahr, N. H. Matlis, V, Leroux, T, Eichner, K. Ravi, H. Ishizuki, T. Taira, F. X. Kärtner abd A. R. Maier, this conference.

[7] L. J. Wong, A. Fallahi and F. X. Kärtner, Opt. Express 21, 9792 (2013).

[8] E. A. Nanni, W. R. Huang, K. Ravi, A. Fallahi, G. Moriena, R. J. Miller, and F. X. Kärtner, Nat. Communications 6, p. 8486 (2015).

[9] W. R. Huang, et al, Optica 3, p. 1209 (2016).

[10] A. Fallahi, M. Fakhari, A. Yahaghi, M. Arrieta, and F. X. Kärtner, Phys. Rev. Accelerators and Beams 19, 081302 (2016).

[11] M. Fakahri, A. Fallahi and F. X. Kärtner, to appear in Phys. Rev. Accelerators and Beams 19, 081302 (2017).

[12] D. Zhang, A. Fallahi, M. Hemmer, X. Wu, M. Fakhari, Y. Hua, H. Cankaya, A.-L. Calendron, L. Zapata, N. H. Matlis, and F. X. Kärtner, arXiv 1711.03024. (2017)

[13] F. X. Kärtner et al. doi:10.1016/j.nima.2016.02.080. 